\title{
Arc-Induced Long-Period Fiber Gratings in the Dispersion Turning Points
}

\author{
Celso Colaço, Paulo Caldas, Ignacio Del Villar, Rui Chibante, and Gaspar Rego
}

\begin{abstract}
We demonstrated the possibility to inscribe longperiod fiber gratings (LPFGs) in a B/Ge codoped fiber by using grating periods shorter than $150 \mu \mathrm{m}$. We also have arc-induced in the SMF 28 fiber an LPFG in the dispersion turning points by using a grating period of $197 \mu \mathrm{m}$. In previous works, the shortest periods were, respectively, of the order of 190 and $320 \mu \mathrm{m}$ for the same fibers. To achieve such a considerable reduction in the grating periods which enables access to the higher order cladding modes (higher sensitivity), we have developed a high-voltage power supply that allows for a constant and stable electric current ranging from 10.5 up to $21 \mathrm{~mA}$. Computer simulations were used to identify the cladding mode resonances for each grating inscribed in the different fibers. The fabricated LPFGs were characterized as a function of the external refractive index from 1.33 up to 1.42 , and an average refractive index sensitivity of $-720 \mathrm{~nm} / \mathrm{RIU}$ in the 1.33-1.41 range was obtained for a 192- $\mu \mathrm{m}$ LPFG without further optimization, such as the use of etching or thin films deposition.
\end{abstract}

Index Terms - Long-period fiber grating, electric arc technique.

\section{INTRODUCTION}

$\mathbf{L}$ ONG-PERIOD fiber gratings (LPFGs) are periodic structures inscribed in a fiber, with periods typically in the range of $100 \mu \mathrm{m}$ to $1 \mathrm{~mm}$ which couples light between the core mode and co-propagating cladding modes at specific resonance wavelengths. The gratings transmission spectra contains a series of attenuation bands centered at these discrete resonance wavelengths, each attenuation band corresponding to coupling of a different cladding mode. The gratings behave as selective filters, where the resonance wavelengths depend on the period of the LPFG and also on physical parameters, such as temperature, strain, external refractive index and bending radius. LPFGs can, therefore, be used as sensors of these parameters [1], [2].

Manuscript received December 17, 2015; revised March 8, 2016 and February 16, 2016; accepted March 8, 2016. Date of publication March 9, 2016; date of current version September 22, 2016. This work was supported by the European Regional Development Fund through the Operational Program for Competitiveness and Internationalization, COMPETE 2020 Program, and by National Funds through the Fundação para a Ciência e a Tecnologia (Portuguese Foundation for Science and Technology) under Project POCI-01-0145-FEDER-006961.

C. Colaço is with the Instituto de Engenharia de Sistemas e Computadores, Tecnologia e Ciência, Porto 4169-007, Portugal (e-mail: celsocolaco@ ultra-wave.com).

P. Caldas and G. Rego are with the Instituto de Engenharia de Sistemas e Computadores, Tecnologia e Ciência, Porto 4169-007, Portugal, and also with the Instituto Politécnico de Viana do Castelo, ESTG, Viana do Castelo 4900-348, Portugal (e-mail: pcaldas@estg.ipvc.pt; gmrego@fc.up.pt).

I. Del Villar is with the Electrical and Electronic Engineering Department, Public University of Navarra, Pamplona 31006, Spain (e-mail: ignacio. delvillar@unavarra.es).

R. Chibante is with the Instituto Superior de Engenharia do Porto, Porto 4200-072, Portugal (e-mail: rmc@isep.ipp.pt).

Color versions of one or more of the figures in this paper are available online at http://ieeexplore.iee.org.

Digital Object Identifier 10.1109/JLT.2016.2540678
Among the different techniques available to inscribe gratings, the electric arc technique is well-established and disseminated all over the world [3]. The interest of the scientific community results from it being a simple, versatile and low cost technique besides enabling the fabrication of gratings in any kind of fiber [4], [5]. Arc-induced gratings have considerable polarization dependent loss which limits their application in the optical communications field, such as is the case of the equalization of EDFAs gain spectrum [6]. On the other hand, they are thermally resistant and can be used as high temperature sensors [7]. It is well-known that higher sensitivity to the different physical parameters can be obtained if they are written in the dispersion turning points (DTP) [8], [9]. At these points, the slope of the phase matching curves, for each cladding mode resonance, reaches its maximum value. On the other hand, near the DTP the slope steeply increases and changes from positive to negative and, for each grating period, there are two resonance wavelengths for each cladding mode. This is due to the dependence on wavelength of the core and cladding effective refractive indices. For a particular grating period, the phase matching condition can be satisfied for more than one resonance wavelength (for the same cladding mode) since as the wavelength increases the effective refractive index of the cladding mode decreases faster than that of the core [8]. A higher sensitivity can be important to increase the temperature sensitivity of sensors to work in cryogenic environments [10] and also to develop high resolution refractometer sensors based on LPFGs in the DTP with thin films in the transition region [11]. During the past decade a lot of effort was focused on the understanding of the mechanisms of grating formation, types of modes excited and ways to increase reproducibility [12][18]. Being the grating formation associated to a thermal effect, it prevented the fabrication of arc-induced LPFGs in the DTP. Recently, Debowska et al. [19] demonstrated the inscription of LPFGs in the DTP by writing LPFGs in the B/Ge co-doped fiber with periods of the order of $190 \mu \mathrm{m}$ and for which they have used more than 200 arc-discharges. Afterwards, Colaço et al. [20] developed a new high voltage power supply to produce very confined arc discharges establishing a minimum period of $148 \mu \mathrm{m}$ in a B/Ge co-doped fiber (LPFG attenuation bands of $4.5 \mathrm{~dB}$ after 142 arc discharges). It should be stressed that the quest for further reducing the grating period is very pertinent when one deals with the fabrication of LPFGs in few-mode fibers $(100-120 \mu \mathrm{m})[21]$ or LPFGs in the DTP written in photonic crystal fibers $(100 \mu \mathrm{m})$ [22]. The same requirements apply to LPFGs assisted surface plasmon resonance (SPR) [23] where grating periods in the range of $110-120 \mu \mathrm{m}$ are also needed. The requirement can only be relaxed in the case of long-range SPR where grating periods of about $200 \mu \mathrm{m}$ may be used [24]. 


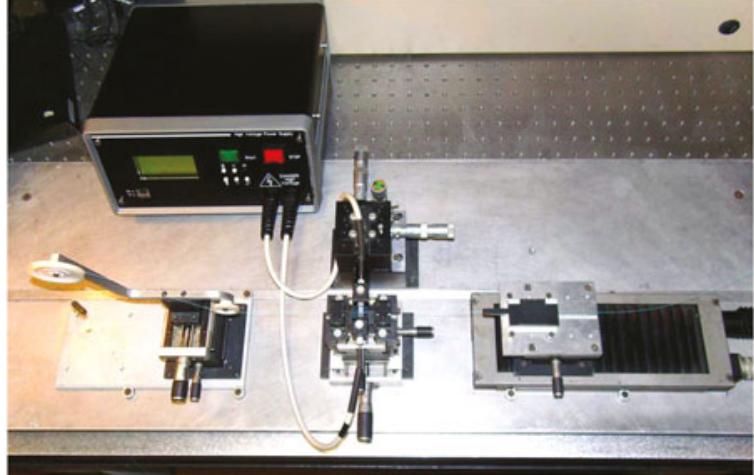

Fig. 1. Experimental set-up: Mechanical arrangement and high voltage power supply.

In this work we describe the whole process used to attain the DTP in the Corning SMF28 fiber and also in the Fibercore (PS 1250/1550) B/Ge co-doped fiber. We have also identified the cladding modes through computer simulations. Section II-A describes the experimental setup, comprising the new high voltage power supply, used to inscribe the gratings in three different fibers. The choice of the fabrication parameters depending on the fiber is also discussed in Section II-B. Section II-C describes the fabrication process of arc-induced gratings in the DTP and results of computer simulations to identify the cladding modes are presented. The influence of the external refractive index on the LPFGs transmission spectra is given in Section II-D.

\section{EXPERIMENTAL RESULTS AND DISCUSSION}

\section{A. Experimental Setup}

The experimental set-up used to fabricate the gratings is shown in Fig. 1. The whole process is computer controlled, being the mechanical arrangement used to control the fiber positioning/displacement established a decade ago [5]. In which concerns the electrical part, the power source used to feed the electrodes was supplied by the BICC AFS1300 fusion splicer [5]. As demonstrated in [17] the produced arc discharges were directional, due to the dc output, and had a large width, as expected for a fusion splicer machine even though the electrodes gap was only of $0.95 \mathrm{~mm}$. Meanwhile, we have developed a new high voltage power source (also shown in Fig. 1) that allows for confined arc discharges. The dimensions of the arc can be further controlled by changing the distance between the electrodes (the shorter the electrodes gap the smaller is the arc width). The ac power source possesses a feedback loop that keeps a constant rms electric current, that is measured by a current sensing shunt resistor. The state of the electrodes can be monitored by using a digital oscilloscope. In Fig. 2 it can be seen a normal arc discharge and a discharge when the electrodes present some degradation. In the latter, to keep the same electric current (see top Fig. 2(a), (b)), a higher voltage peak (see bottom Fig. 2(b)) for the arc ignition is required. That is, the electrodes oxidation demands for a higher voltage in order to establish the arc through air.

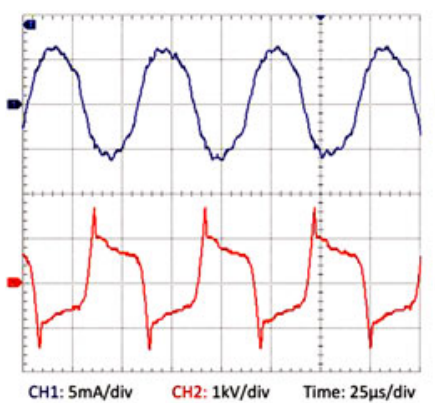

(a)

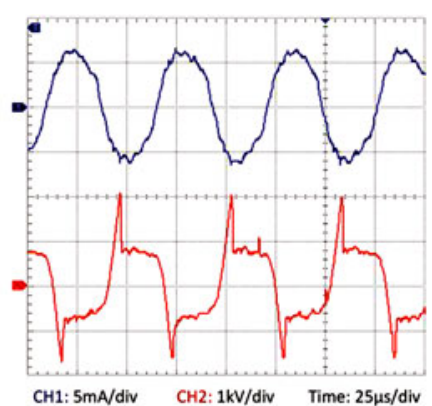

(b)
Fig. 2. (a) Normal arc discharge: electric current (top) and voltage (bottom). (b) "Degraded" arc discharge.

Gratings were inscribed in the SMF28 fiber and in two PS 1250/1550 B/Ge co-doped fibers from Fibercore (fiber \#1: NA $=0.13 ; \mathrm{MFD}=9.6 \mu \mathrm{m} ; \lambda_{\text {cut }-\mathrm{off}}=1150 \mathrm{~nm}$ and fiber \#2: $\left.\mathrm{NA}=0.14 ; \mathrm{MFD}=8.9 \mu \mathrm{m} ; \lambda_{\text {cut }- \text { off }}=1238 \mathrm{~nm}\right)$ with grating periods ranging from 148 up to $540 \mu \mathrm{m}$ (fabrication parameters: electric current of 11-18 mA; 200-700 ms arc duration; 2-5 g pulling weight; 20-400 arc discharges).

\section{B. Optimization of the Fabrication Parameters}

For this new high voltage power supply and for the sake of comparison with a well-known reference, we had to adjust the fabrication parameters in order to reproduce the spectrum of a grating inscribed in the SMF28 fiber with a period of $540 \mu \mathrm{m}$, obtained previously by using the BICC AFS1300 fusion splicer as a power source [17]. We obtained a similar spectra after 27 arc discharges using an electric current of $15 \mathrm{~mA}$ during $200 \mathrm{~ms}$. To monitor the gratings spectra during their inscription we have used a white light source (WLS), from Ocean Optics HL-2000HP, to illuminate the LPFGs and their transmission spectra were registered with an optical spectrum analyzer, from Yokogawa AQ6370C, with a resolution of $1 \mathrm{~nm}$. The presented spectra are normalized to the spectrum of the WLS, taken 10 min after temperature stabilization. Fig. 3 shows the spectra of several gratings with a period of $400 \mu \mathrm{m}$ written in the SMF28 fiber using different fabrication parameters (electrodes gap and external tension were set to $0.9 \mathrm{~mm}$ and $5.2 \mathrm{~g}$, respectively). As can be seen, the increase of the electric current and arc duration lead to an increase of the resonances coupling strength and also to a slight displacement towards longer wavelengths. The former is a well-known result but the latter is in contradiction with previous studies [25]. This may be due to the fact that in this setup, a smaller width of the arc discharge leads to a less effective stress relaxation of the cladding and, therefore, the change in the core refractive index prevails over that of the cladding. Then, we changed the fabrication parameters in order to inscribe gratings with shorter periods. To reach periods of about $200 \mu \mathrm{m}$ we had to displace the fiber to a lower temperature region and higher temperature gradient, by down-shift the fiber relatively to the arc [16], [17]. This procedure reinforces coupling to asymmetric cladding modes as shown by simulations in Fig. 7(a). Fig. 4 shows the spectra of gratings with different periods: 276, 253, 


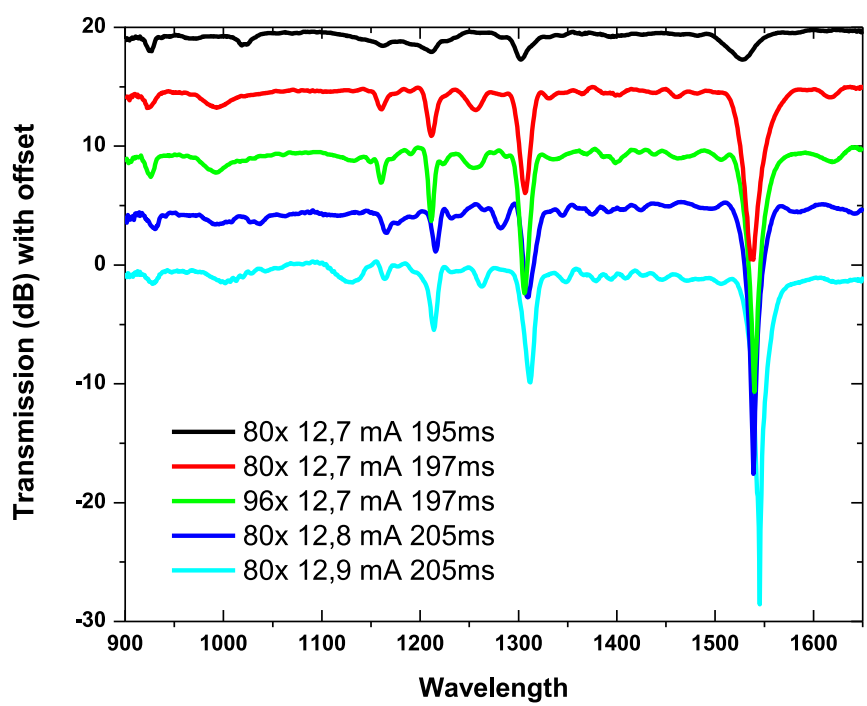

Fig. 3. Influence of the fabrication parameters on the spectra of $400 \mu \mathrm{m}$ gratings arc-induced in the SMF28 fiber.

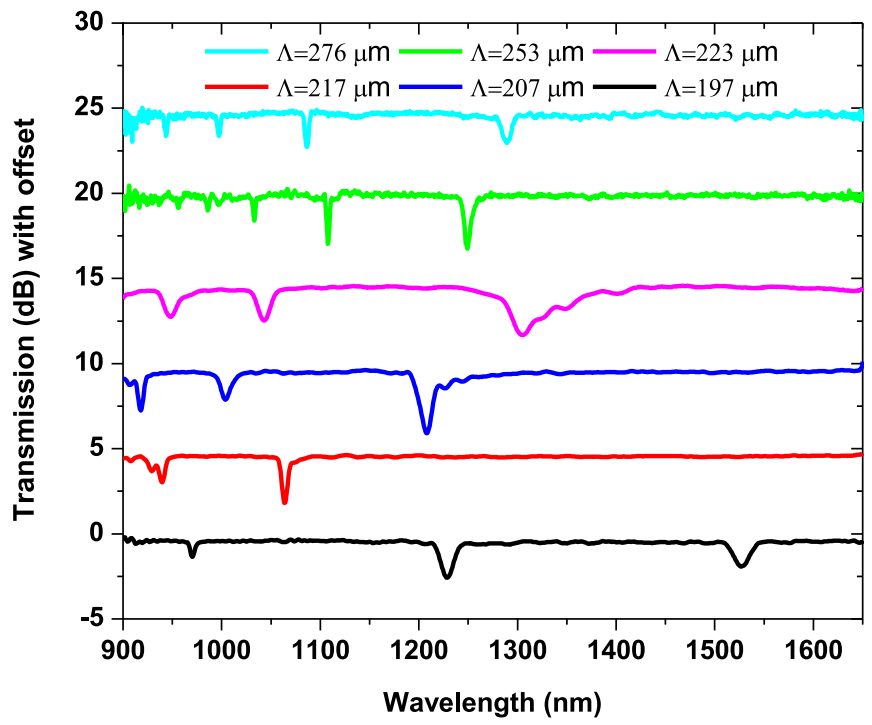

Fig. 4. Spectra of gratings arc-induced in the SMF28 fiber.

223, 217, 207 and $197 \mu \mathrm{m}$ (the first two written with 260 arc discharges and the other with 400 arc discharges and an external tension of $2 \mathrm{~g}$ ). The other fabrication parameters were, respectively, $11 \mathrm{~mA}$ and $280 \mathrm{~ms}, 18.1 \mathrm{~mA}$ and $118 \mathrm{~ms}, 14 \mathrm{~mA}$ and $370 \mathrm{~ms}$ for the next two gratings, and $12.7 \mathrm{~mA}$ and $580 \mathrm{~ms}$ for the last two gratings. It should be stressed that we obtained strong gratings for periods above $350 \mu \mathrm{m}$ while for periods around $200 \mu \mathrm{m}$ only weak gratings ( $4 \mathrm{~dB}$ attenuation loss peaks) were attained.

Afterwards we inscribed gratings in the $\mathrm{B} / \mathrm{Ge}$ co-doped fibers. We noted that for these fibers lower electric current values, arc duration and number of arc discharges were required in order to produce the gratings. In the case of the fiber \#1, the fabrication parameters used were $13.8 \mathrm{~mA}, 308-320 \mathrm{~ms}$ and arc discharges ranging from 140 up to 170 (see Fig. 5(a)). In the case of the fiber \#2, the optimum fabrication parameters were $12.7 \mathrm{~mA}$,

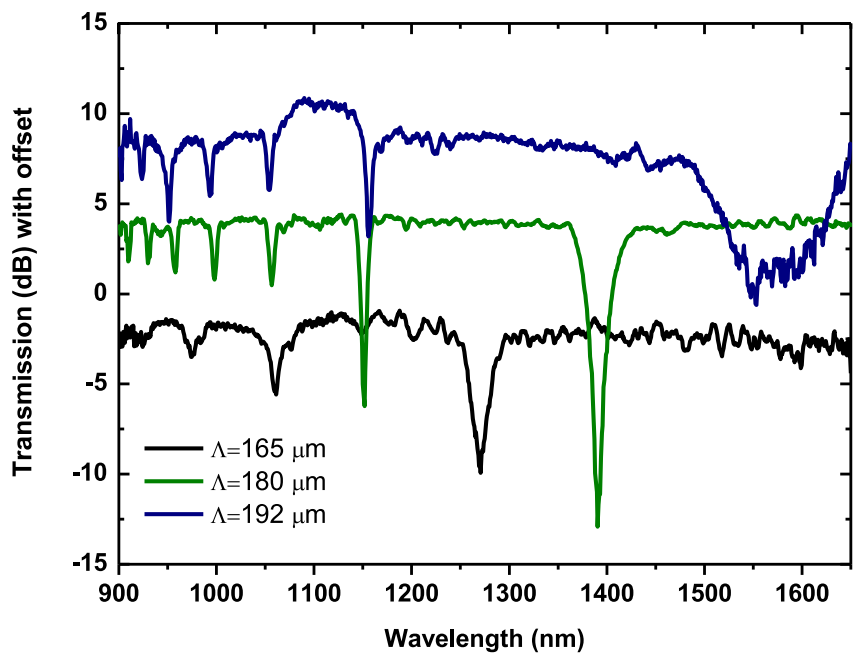

(a)

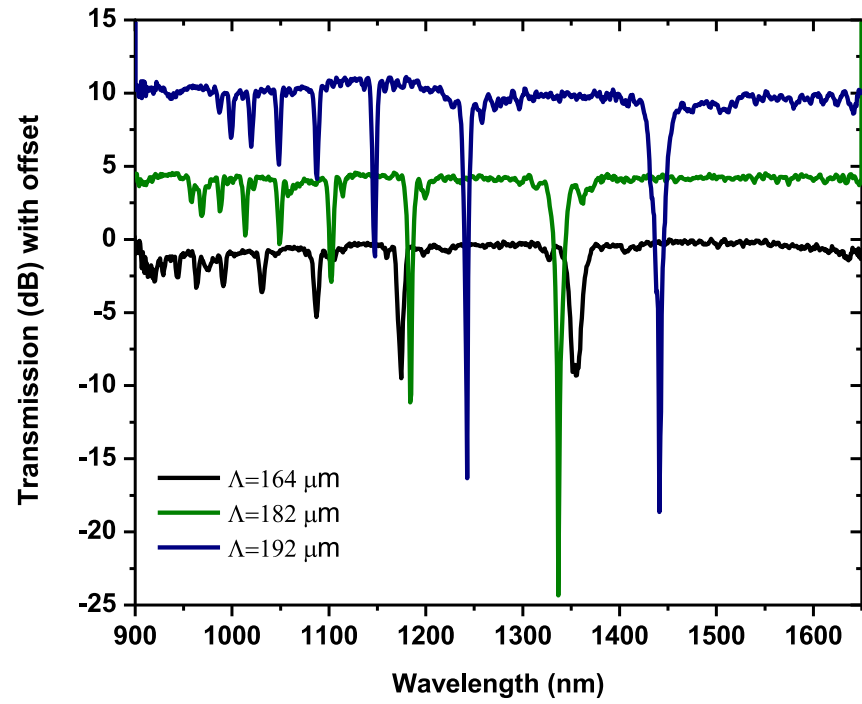

(b)

Fig. 5. Transmission spectra of LPFGs, inscribed in the B/Ge co-doped fibers: (a) fiber \#1 and (b) fiber \#2.

660-680 ms and 122 arc discharges (see Fig. 5(b)). Then, for the sake of comparison, we have also used the same fabrication parameters to inscribe gratings in a short piece remaining from the first fiber $(180 \mu \mathrm{m}-\mathrm{LPFG}$ in Fig. 5(a)). It should be noted that these fibers are two batches of the same Fibercore fiber but unfortunately they had different physical properties as described previously. Fig. 5 shows the spectra of several gratings written in both fibers, for which strong gratings were produced with only 122 arc discharges. However, the resonance wavelengths are different when the same grating periods are used. Consequently, the DTP is found for a different period, as we will see in the next section.

\section{Gratings Fabrication in the DTPs}

The fabrication parameters were optimized in order to be possible to write the gratings in the DTP. In particular we have decreased the electrodes gap (from 1.0 to $0.9 \mathrm{~mm}$ ). A LPFG 


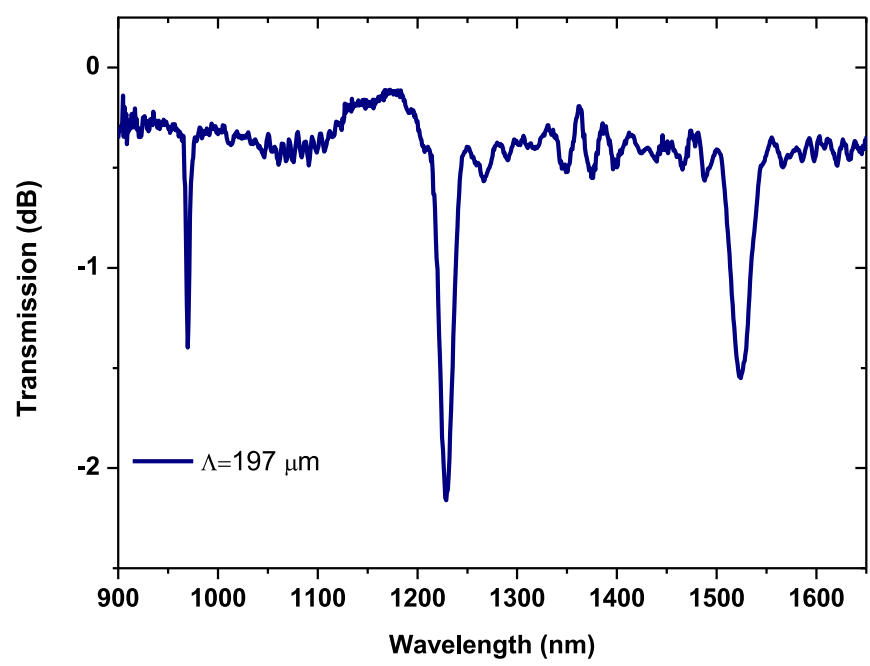

(a)

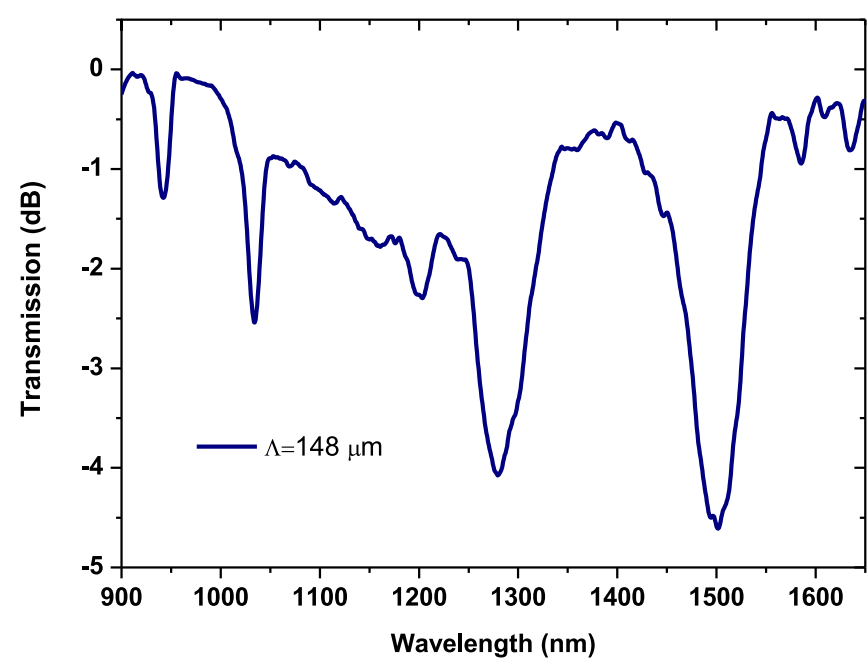

(b)

Fig. 6. Transmission spectra of LPFGs near the DTP, inscribed in the (a) SMF28 fiber and (b) B/Ge co-doped fiber \#1.

was inscribed in the SMF28 fiber with a period of $197 \mu \mathrm{m}$ and its spectrum is shown in Fig. 6(a). This LPFG is in the vicinity of the DTP since when surrounded by water the two resonances moves in opposite ways (see Fig. 8(a)). The fabrication parameters were: electric current of $12.7 \mathrm{~mA}, 600 \mathrm{~ms}$ arc duration, $2 \mathrm{~g}$ pulling weight and 400 arc discharges. It should be stressed that we are working in the limits of the electric arc technique since it was only possible to write a weak grating even after 400 arc discharges. Even so, this result is quite impressive since previously in this fiber the shortest period was larger than $300 \mu \mathrm{m}$ [26]. Fig. 6(b) shows the arc-induced LPFG with the shortest grating period $148 \mu \mathrm{m}$. This grating was inscribed in fiber \#1, from Fibercore, using the following fabrication parameters: electric current of $13.8 \mathrm{~mA}, 308 \mathrm{~ms}$ arc duration, $2 \mathrm{~g}$ pulling weight and 142 arc discharges. Since the mechanism of grating formation is different in this fiber [15] we believe that further optimization is still possible, in particular by changing the relative position between the fiber and the arc discharge. In any case we decreased the shortest period from about $190 \mu \mathrm{m}$ [18] to less than $150 \mu \mathrm{m}$ and we have also reduced in about half the required number of arc discharges.

In order to identify the cladding modes involved in these gratings we have simulated the dispersion curves for gratings arc-induced in these fibers. The modified phase matching condition has been used [27]. This permits to obtain the resonance wavelengths with an error lower than $0.1 \%$ :

$$
\beta_{01}(\lambda)+s_{0} \zeta_{01,01}(\lambda)-\left(\beta_{0 j}(\lambda)+s_{0} \zeta_{0 j, 0 j}(\lambda)\right)=\frac{2 \pi N}{\Lambda},
$$

where $\beta_{01}$ and $\beta_{0 j}$ are the propagation constants of the core and the $j$ cladding modes respectively, $\Lambda$ is the period of the grating, $\zeta_{01,01}$ and $\zeta_{0 j, 0 j}$ are the self-coupling coefficients of the core and the $j$ cladding modes, $s_{0}$ is the coefficient of the first Fourier component of the grating and $N$ is the diffraction order. For asymmetric coupling, $1, j$ cladding modes must be considered [16], [27]:

$$
\beta_{01}(\lambda)+s_{0} \zeta_{01,01}(\lambda)-\left(\beta_{1 j}(\lambda)+s_{0} \zeta_{1 j, 1 j}(\lambda)\right)=\frac{2 \pi N}{\Lambda} .
$$

The cladding index was modeled with the optical fiber cladding, made of fused silica, has been estimated with the well-known Sellmeier equation:

$$
n^{2}(\omega)=1+\sum_{j=1}^{m} \frac{B_{j} \omega_{j}^{2}}{\omega_{j}^{2}-\omega^{2}}
$$

with parameters: $B_{1}=0.691663, B_{2}=0.4079426, B_{3}=$ $0.8974794, \lambda_{1}=0.0684043 \mu \mathrm{m}, \lambda_{2}=0.1162414$, and $\lambda_{3}=$ 9.896161, where $\lambda j=2 \pi \mathrm{c} / \omega j$ and $c$ is the speed of light in vacuum [28].

The optical fiber core refractive index of SMF28 for the simulations has been obtained, according to the specifications from Corning, Inc., by increasing the refractive index of the cladding $0.357 \%$. In order to obtain an optimized fit of the experimental and theoretical results, it was necessary to consider that an increase in the cladding refractive index was induced with the arc discharge and this effect depends on the grating period. For higher grating periods the effect was less important than for lower grating periods.

For the $\mathrm{B} / \mathrm{Ge}$ co-doped fibers this fitting was not necessary. The same cladding index was used and the increase of the core refractive index was $0.408 \%$ for $\mathrm{B} / \mathrm{Ge} \# 1$ and $0.47 \%$ for $\mathrm{B} / \mathrm{Ge}$ \#2. A dispersion of the core index was also included for an optimized fitting.

Fig. 7(a) shows the dispersion curves for the LPFGs written in the SMF28 fiber where coupling to asymmetric cladding modes were obtained $\left(\mathrm{LP}_{1, j} ; j \geq 1\right)$. The dispersion curves corresponding to LPFGs inscribed in the $\mathrm{B} / \mathrm{Ge}$ co-doped fibers are presented in Fig. 7(b) and (c). In this case, coupling occurs from the core mode to symmetric cladding modes $\left(\mathrm{LP}_{0, j} ; j \geq 2\right)$. For B/Ge \#1, those gratings that were fabricated with different parameters (one of the LPFGs spectra was previously shown in Fig. 5(a)) do not fit exactly the numerical results but are also included for the sake of comparison. Furthermore, they are a warning for those who deals with simulations. In case of $\mathrm{B} / \mathrm{Ge}$ \#2, it should be stressed that it is possible to reach the DTP, for a 


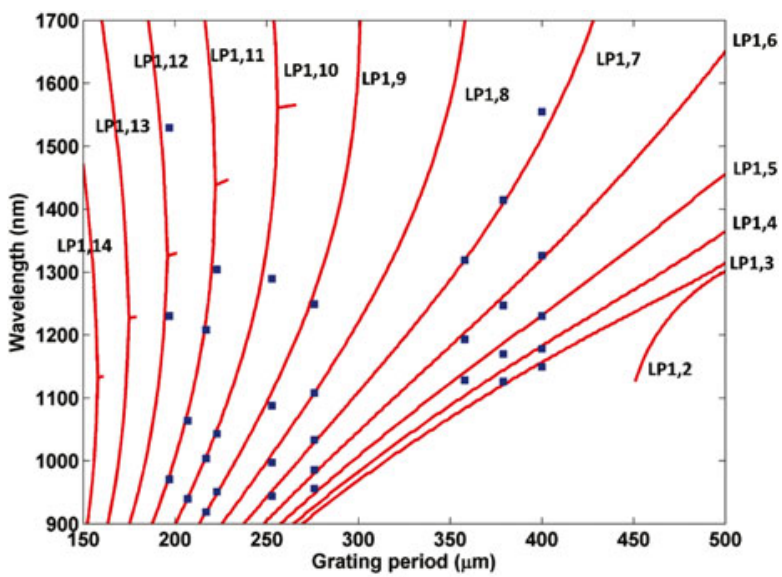

(a)

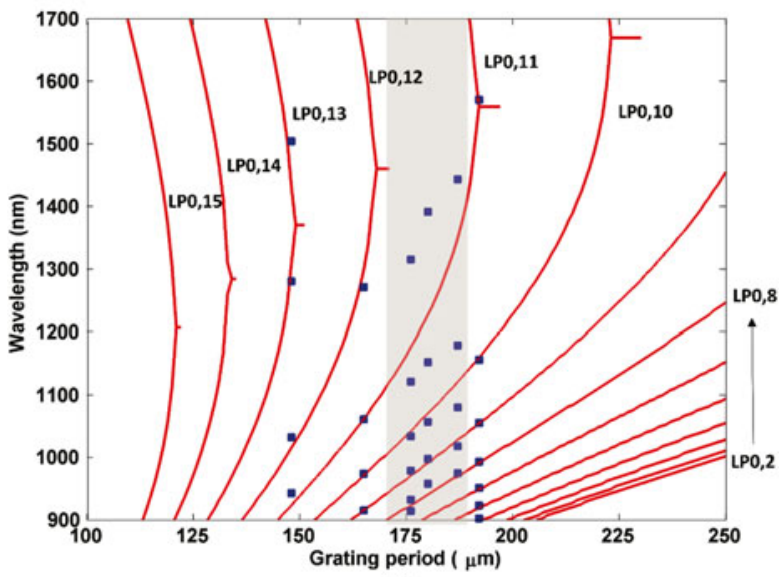

(b)

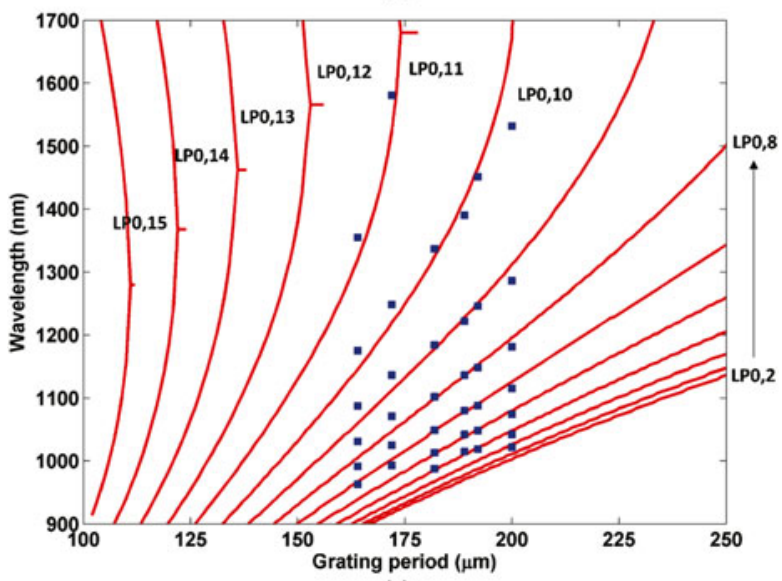

(c)

Fig. 7. Resonance wavelengths for several cladding modes versus grating period for LPFGs, inscribed in the (a) SMF28 fiber, (b) B/Ge co-doped fiber $\# 1$ and (c) B/Ge co-doped fiber \#2. Solid lines represent numerical results and square points experimental results.

resonance at around $1550 \mathrm{~nm}$, by further decreasing the period to about $150 \mu \mathrm{m}$, while in the first fiber we reached it with a period of $192 \mu \mathrm{m}$.

\section{Sensitivity to External Refractive Index}

In order to assess the sensitivity to the surrounding refractive index, several LPFGs were submitted to changes of the external

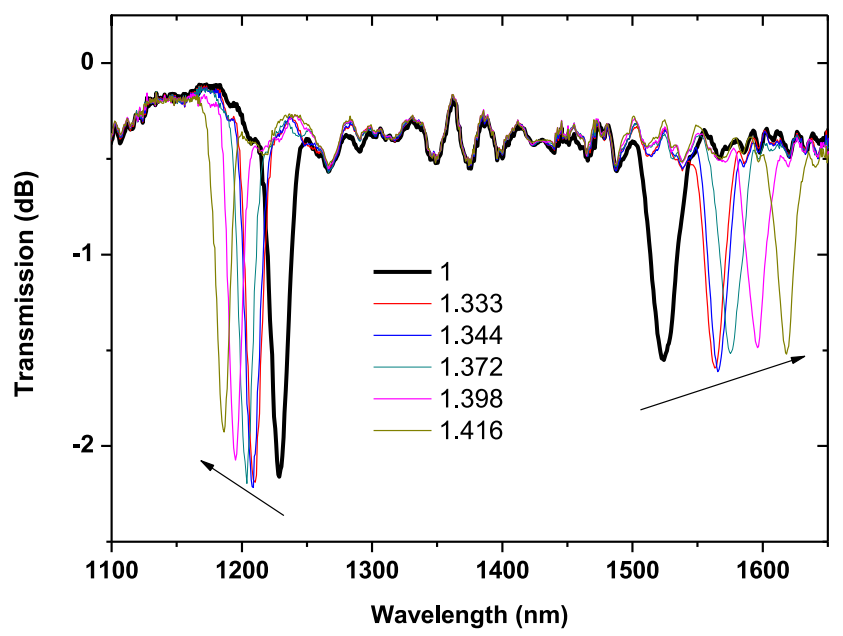

(a)

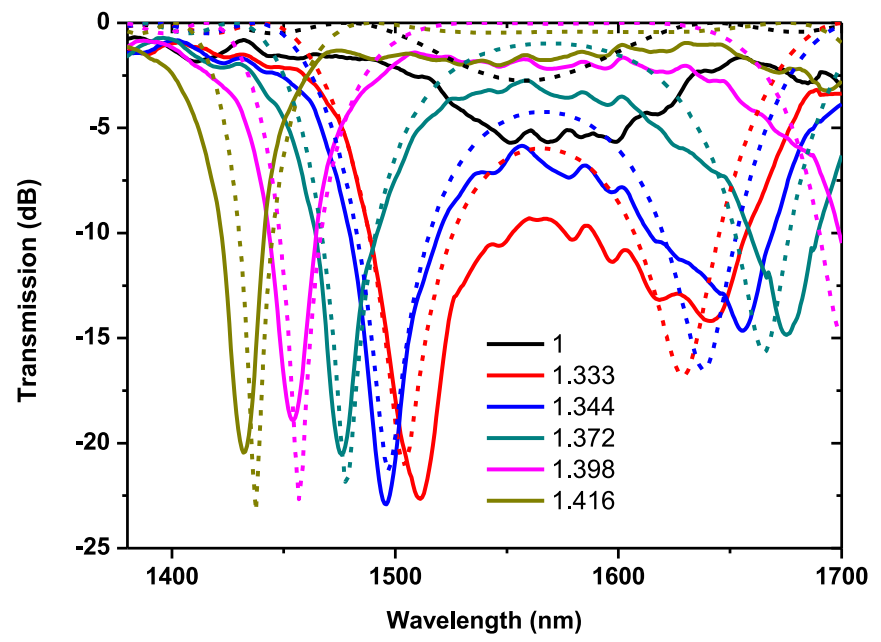

(b)

Fig. 8. Transmission spectra as a function of the external refractive index for several LPFGs in the DTP, inscribed in the (a) SMF28 fiber with $\Lambda=197 \mu \mathrm{m}$; and (b) $\mathrm{B} / \mathrm{Ge}$ co-doped fiber with $\Lambda=192 \mu \mathrm{m}$ (numerical results represented by dashed lines are included).

refractive index in the range 1.33 to 1.42 . To this purpose, each LPFG was glued on a special designed cuvette [29] which keeps the fiber under a constant tension and avoids bending of the grating. The LPFG transmission spectra were recorded, by using the optical arrangement described in Section II-B, after immersing the fiber in samples of water combined with different percentages of ethylene glycol. The refractive index of the different samples was calibrated using an Abbe refractometer with the sodium D line $(589 \mathrm{~nm})$. To avoid contamination in-between measurements, the fiber was cleaned with alcohol and dried naturally. It should be stressed that any environmental temperature change occurring during the $20 \mathrm{~min}$ of the experiment has a negligible effect on the temperature dependence of the refractive index of water-glycol mixtures [29]. This was confirmed by full recovering of the LPFG transmission spectrum in water, recorded after the experiment.

Fig. 8(a) shows the spectrum of a $197 \mu \mathrm{m}-\mathrm{LPFG}$, inscribed in the SMF28 fiber in the vicinity of the DTP, as a function 


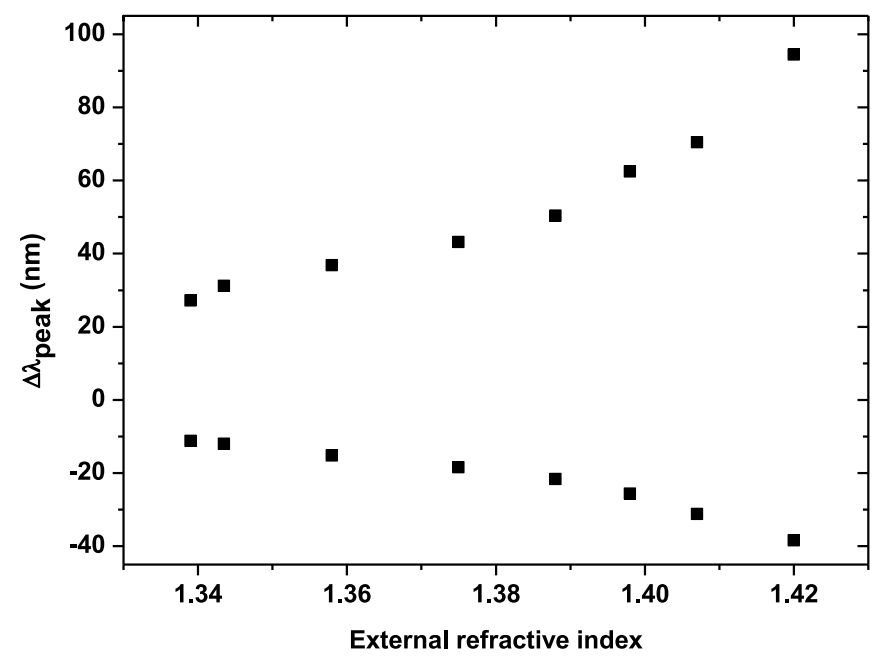

(a)

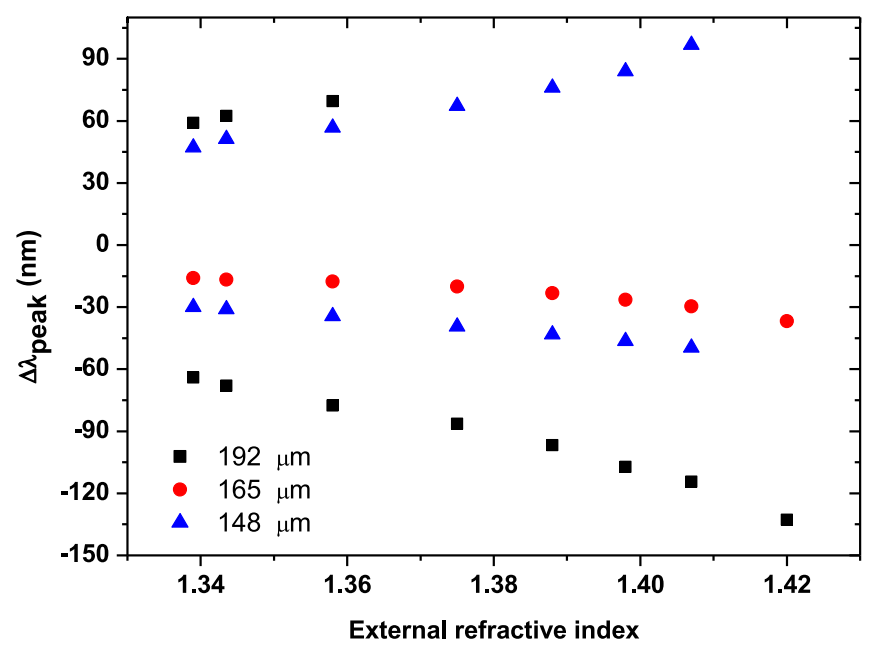

(b)

Fig. 9. Sensitivity to changes in the external refractive index of LPFGs near the DTP, inscribed in the (a) SMF28 fiber $(\Lambda=197 \mu \mathrm{m})$; and (b) B/Ge co-doped fiber \#1.

of the surrounding refractive index. As it can be observed, as the refractive index of the external medium increases, the resonances belonging to the same cladding mode moves in opposite directions being the sensitivity larger for the one at longer wavelengths. This is a consequence of the larger slope of the resonance wavelength versus grating period curve above the DTP [4]. A similar behavior can be observed in Fig. 8(b) although in this case the $192 \mu \mathrm{m}$-LPFG is written in the $\mathrm{B} / \mathrm{Ge}$ co-doped fiber in the DTP as is identified by the single resonance in air that afterwards splits in two when the grating is surrounded by water and water-glycol mixtures. Numerical results following the model used in Fig. 7(b) have been added to Fig. 8(b) (dashed lines), showing a good agreement with the experimental results. The refractive index used is reduced in 0.012 refractive index units (RIU) due to dispersion of glycol and water in the infrared region [30], [31].

Fig. 9 shows the refractive index sensitivity for several gratings, with different periods, inscribed in the two fibers. As it is well-known the sensitivity increases as one approaches the cladding refractive index [9], nevertheless we present results for practical aqueous solutions (1.33 is the water RI). In the case of Fig. 9(a), the result corresponds to the highest value obtained for an LPFG written in the SMF28 fiber, near the DTP. The sensitivity for the resonance at longer wavelengths is about two times higher ( $590 \mathrm{~nm} / \mathrm{RIU}$ in the 1.33-1.41 RI range) than for the resonance at shorter wavelengths and is also two times higher than the value obtained for a $345 \mu \mathrm{m}$-LPFG [26]. As can be seen in Fig. 9(b), the highest sensitivity was obtained for the $192 \mu \mathrm{m}$-LPFG written in the DTP in the B/Ge co-doped fiber $\# 1$, that is, a value of $-720 \mathrm{~nm} / \mathrm{RIU}$ in the $1.33-1.41 \mathrm{RI}$ range, for the resonance at shorter wavelengths (a value of $675 \mathrm{~nm} / \mathrm{RIU}$ in the 1.33-1.41 RI range was also obtained for the $148 \mu \mathrm{m}$ LPFG, for the resonance at longer wavelengths). It should be noted that the results presented for the $148 \mu \mathrm{m}-\mathrm{LPFG}$, for the resonance at shorter wavelengths, and for $192 \mu \mathrm{m}-\mathrm{LPFG}$, for the resonance at longer wavelengths, have some uncertainty due to noise in the experimental data as can be observed, for instance, in Fig. 8(b). In any case, by comparison of the $197 \mu \mathrm{m}-\mathrm{LPFG}$, written in the SMF28 fiber, and the $192 \mu \mathrm{m}-\mathrm{LPFG}$, written in the $\mathrm{B} / \mathrm{Ge} \# 1$ fiber, the sensitivity obtained for the resonance at shorter wavelengths (cladding modes: $\mathrm{LP}_{1,11}$ versus $\mathrm{LP}_{0,11}$ ) is three times higher for the grating written in the $\mathrm{B} / \mathrm{Ge}$ fiber. The higher sensitivity obtained for gratings written in this fiber is in accordance with previous works [9], [32].

\section{CONCLUSION}

We have developed a high voltage power supply that permitted us to arc-induce LPFGs, in the DTP, in two B/Ge codoped fibers and in one SMF28 fiber by using grating periods shorter than respectively, 150 and $200 \mu \mathrm{m}$. We have identified the cladding modes involved in the coupling by computer simulations. The fabricated LPFGs were characterized as a function of the external refractive index from 1.33 up to 1.42 . Further work is still required in order to increase the coupling strength of LPFGs in the DTP, inscribed in the SMF28 fiber, which in turn will allow the development of practical sensors. Currently, we are optimizing the fabrication of LPFGs in the B/Ge co-doped fibers, in the DTP, with high sensitivity to be used at cryogenic temperatures and also as refractometer sensors comprising thin films in the transition region [33].

\section{REFERENCES}

[1] A. M. Vengsarkar, P. J. Lemaire, J. B. Judkins, V. Bhatia, T. Erdogan, and J. E. Sipe, "Long-period fiber gratings as band-rejection filters," J. Lightw. Technol., vol. 14, no. 1, pp. 58-65, Jan. 1996.

[2] V. Bathia, "Applications of long-period gratings to single and multiparameter sensing," Opt. Exp., vol. 4, no. 11, pp. 457-466, May 1999.

[3] G. Rego, "Arc-induced long period fiber gratings," J. Sens., vol. 2016, Jan. 2016, Art. No. 3598634.

[4] G. Rego, O. Okhotnikov, E. M. Dianov, and V. B. Sulimov, "Hightemperature stability of long-period fiber gratings using an electric arc," J. Lightw. Technol., vol. 19, no. 10, pp. 1574-1579, Oct. 2001.

[5] G. Rego, P. V. S. Marques, J. L. Santos, and H. M. Salgado, "Arc-induced long-period gratings," Fiber Integr. Opt., vol. 24, nos. 3/4, pp. 245-259, May 2005.

[6] G. Rego, J. L. Santos, and H. M. Salgado, "Polarization dependent loss of arc-induced long-period fibre gratings," Opt. Commun., vol. 262, no. 2, pp. 152-156, Jun. 2006. 
[7] G. Rego, P. Caldas, O. Ivanov, and J. L. Santos, "Investigation of long term stability of arc-induced gratings heat treated at high temperatures," Opt. Commun., vol. 284, no. 1, pp. 169-171, Jan. 2011.

[8] V. Grubsky and J. Feinberg, "Long-period fiber gratings with variable coupling for real-time sensing applications," Opt. Lett., vol. 25, no. 4, pp. 203-205, Feb. 2000.

[9] X. Shu, L. Zhang, and I. Bennion, "Sensitivity characteristics of longperiod fiber gratings," J. Lightw. Technol., vol. 20, no. 2, pp. 255-266, Feb. 2002.

[10] R. Martins, P. Caldas, B. Teixeira, J. Azevedo, J. Monteiro, J. H. Belo, J. P. Araújo, J. L. Santos, and G. Rego, "Cryogenic temperature response of reflection-based phase-shifted long-period fiber gratings," J. Lightw. Technol., vol. 33, no. 12, pp. 2511-2517, Jun. 2015.

[11] G. Rego, "A review of refractometric sensors based on long period fiber gratings," Sci. World J., vol. 2013, Dec. 2013, Art. No. 913418.

[12] G. Rego, L. M. M. B. F. Santos, B. Schröder, P. V. S. Marques, J. L. Santos, and H. M. Salgado, "In situ temperature measurement of an optical fiber submitted to electric arc discharges," IEEE Photon. Technol. Lett., vol. 16 , no. 9, pp. 2111-2113, Sep. 2004.

[13] F. Dürr, G. Rego, P. V. S. Marques, S. L. Semjonov, E. M. Dianov, H. G. Limberger, and R. P. Salathé, "Tomographic stress profiling of arcinduced long period fiber gratings," J. Lightw. Technol., vol. 23, no. 11, pp. 3947-3953, Nov. 2005.

[14] A. Malki, G. Humbert, Y. Ouerdane, A. Boukhenter, and A. Boudrioua, "Investigation of the writing mechanism of electric-arc-induced long-period fiber gratings," Appl. Opt., vol. 42, no. 19, pp. 3776-3779, Jul. 2003.

[15] K. Morishita and Y. Miyake, "Fabrication and resonance wavelengths of long-period gratings written in a pure-silica photonic crystal fiber by the glass structure change," J. Lightw. Technol., vol. 22, no. 2, pp. 625-630, Feb. 2004.

[16] G. Rego, O. Ivanov, and P. V. S. Marques, "Demonstration of coupling to symmetric and antisymmetric cladding modes in arc-induced long-period fiber gratings," Opt. Exp., vol. 14, no. 21, pp. 9594-9599, Oct. 2006.

[17] O. Ivanov and G. Rego, "Origin of coupling to antisymmetric modes in arc-induced long-period fiber gratings," Opt. Exp., vol. 15, no. 21 pp. 13936-13941, Oct. 2007.

[18] L. A. García-de-la-Rosa, I. Torres-Gómez, A. Martínez-Ríos, D. MonzónHernández, and J. Reyes-Gómez, "Background loss minimization in arcinduced long-period fiber gratings," Opt. Eng., vol. 49, no. 6, Jun. 2010, Art. No. 065001

[19] A. K. Debowska, M. Smietana, P. Mikulic, and W. J. Bock, "High temperature nano-coated electric-arc-induced long-period gratings working at the dispersion turning point for refractive index sensing," Jpn. J. Appl. Phys., vol. 53, Jul. 2014, Art. No. 08ME01.

[20] C. Colaço, P. Caldas, R. Chibante, and G. Rego, "Arc-induced gratings in the turning points," in Proc. 24th Int. Conf. Opt. Fiber Sens. SPIE, Jun. 2015, vol. 9634, Art. No. 96346K-1.
[21] S. Ramachandran, "Dispersion-tailored few-mode fibers: A versatile platform for in-fiber photonic devices," J. Lightw. Technol., vol. 23, no. 11, pp. 3426-3443, Nov. 2005

[22] J. Kanka, "Long-period gratings in photonic crystal fibers operating near the phase-matching turning point for evanescent chemical and biochemical sensing," Proc. SPIE, vol. 8370, pp. 837003-1-837003-11, 2012.

[23] T. Shuster, R. Herschel, N. Neumann, and C. G. Schaffer, "Miniaturized long-period fiber grating assisted surface plasmon resonance sensor," J. Lightw. Technol., vol. 30, no. 8, pp. 1003-1008, Apr. 2012.

[24] Q. Liu and K. S. Chiang, "Refractive-index sensor based on long-range surface plasmon mode excitation with long period waveguide grating," Opt. Exp., vol. 17, no. 10, pp. 7933-7942, Apr. 2009.

[25] G. Rego, "Arc-induced long-period fibre gratings: Fabrication and their applications in optical communications and sensing [dissertation]," $\mathrm{Ph} . \mathrm{D}$ dissertation, Dept. Electr. Comput. Eng., Univ. Porto, Porto, Portugal, 2006

[26] M. Smietana, W. J. Bock, and P. Mikulic, "Increasing sensitivity of arcinduced long-period gratings-Pushing the fabrication technique toward its limits," Meas. Sci. Technol., vol. 22, pp. 115-203, Jan. 2011.

[27] E. Anemogiannis, E. N. Glytsis, and T. K. Gaylord, "Transmission characteristics of long-period fiber gratings having arbitrary azimuthal/radial refractive index variation," J. Lightw. Technol., vol. 21, no. 1, pp. 218-227, Jan. 2003.

[28] I. H. Malitson, "Interspecimen comparison of the refractive index of fused silica,” J. Opt. Soc. Am., vol. 55, no. 10, pp. 1205-1209, Oct. 1965.

[29] D. Pereira, O. Frazão, and J. L. Santos, "Fibre Bragg grating sensing system for simultaneous measurement of salinity and temperature," Opt. Eng., vol. 43, no. 2, pp. 299-304, Feb. 2004

[30] D. Masahiko and A. Masumura, "Measurement of the refractive index of distilled water from the near-infrared region to the ultraviolet region," Appl. Opt., vol. 46, no. 18, pp. 3811-3820, Jun. 2007.

[31] P. R. Cooper, "Refractive-index measurements of liquids used in conjunction with optical fibers," Appl. Opt., vol. 22, no. 19, pp. 3070-3072, Oct. 1983.

[32] V. Guzman-Ramos, D. E. Ceballos-Herrera, and R. Selvas-Aguilar, "Numerical analysis of $\mathrm{GeO}_{2}$-concentration effects in arc-induced long-period fiber gratings under external refractive-index changes," Opt. Rev., vol. 21, no. 2, pp. 143-149, Feb. 2014.

[33] I. D. Villar, "Ultrahigh-sensitivity sensors based on thin-film coated long period gratings with reduced diameter, in transition mode and near the dispersion turning point," Opt. Exp., vol. 23, no. 7, pp. 8389-8398, Apr. 2015.

Authors' biographies not available at the time of publication. 